\title{
Proactive telephone peer support to help pregnant women stop smoking
}

\author{
Laura J Solomon, Roger H Secker-Walker, Brian S Flynn, Joan M Skelly, Eleanor L Capeless
}

Department of

Psychology, University of Vermont, Burlington, Vermont, USA

L J Solomon

Office of Health Promotion Research, University of Vermont R H Secker-Walker B S Flynn

Biometry Facility, University of Vermont J M Skelly

Department of Obstetrics and Gynecology, University of Vermont E L Capeless

Correspondence to: Laura J Solomon, PhD Department of Psychology, Dewey Hall, University of Vermont, Burlington, VT 05405, USA;

Lsolomon@zoo.uvm.edu
Most strategies to help pregnant women stop smoking have relied on cessation advice provided by health care professionals during prenatal visits and/or printed materials designed to encourage self quitting. ${ }^{1}$ Two meta-analyses of smoking cessation studies conducted within health care facilities ${ }^{12}$ revealed that repeated contact with multiple providers (physicians and non-physicians) in multiple formats (face-to-face, telephone, printed material) resulted in better cessation outcomes than single intervention offerings.

The current study built upon this earlier research and tested the impact of physician/ midwife advice to stop smoking accompanied by printed materials with and without proactive telephone peer support provided by a woman ex-smoker between routine prenatal visits. The provision of proactive support, initiated by the support person, has shown promising results with non-pregnant smokers. ${ }^{3}$ A meta-analysis of 13 studies comparing cessation rates for proactive telephone support versus controls revealed a modest significant effect for proactive telephone support. ${ }^{4}$ The combined approach of health professional advice plus proactive telephone peer support enables the professional to legitimise concerns about smoking while permitting the woman ex-smoker to assist the pregnant woman in accomplishing her smoking change goals. Furthermore, the provision of support by telephone bypasses traditional barriers to assistance (for example, cost, transportation, child care) and enables the support to occur frequently and repeatedly. Finally, referral to proactive telephone support requires little time, making it a practical way to support smoking cessation activities in a busy practice setting. This study was designed to test the impact of proactive telephone peer support added to physician/midwife advice to help pregnant women stop smoking.

\section{Methods}

Participants were 151 pregnant women who reported smoking at least one cigarette in the past week when screened at their first prenatal visit to the largest obstetric practice in Vermont during 1996-97. Women smokers in this practice tended to be white, English speaking, and of lower income and education. Pregnant women smokers were approached by a nurse interviewer who described the study and obtained the woman's consent to participate. The refusal rate was $19 \%$. Consenting women responded to a baseline questionnaire read aloud by the interviewer. This questionnaire assessed demographics, smoking history, current smoking behaviour, smoking environment, and stage of readiness to change. Participants were randomised into either the experimental or comparison condition. Women in the comparison condition $(\mathrm{n}=74)$ received brief smoking cessation advice consistent with the Agency for Health Care Policy and Research clinical practice guidelines $^{5}$ delivered by an obstetrician/ midwife at the first three prenatal visits along with stage appropriate printed materials. Women in the experimental condition $(n=77)$ received the same advice and materials plus the offer of telephone peer support for women with moderate or high intentions of quitting smoking during their pregnancy.

All obstetricians and midwives were trained to deliver the brief smoking cessation advice in a 45 minute session. The brief counselling legitimised concern about smoking, elicited feelings about quitting, and encouraged progress toward change including setting a quit date. The nurse interviewer placed a protocol prompt sheet on the woman's chart at each of the first three prenatal visits to guide the obstetrician/midwife in delivery of stage appropriate advice. Women in the experimental condition who reported at any of their first three visits that they possibly, probably, or definitely intended to quit smoking during their pregnancy were offered the telephone peer support by the obstetrician/midwife.

The proactive telephone peer support was provided by a woman ex-smoker who received eight hours of training. The support person called the participant within several days of the referral, explained her role, and provided encouragement, guidance, and much reinforcement for positive changes in the woman's smoking. Ongoing calls typically occurred on a weekly basis, but more frequently around a quit date, and less frequently as smoking changes stabilised. On average, the calls lasted 10 minutes, and the woman could elect to stop receiving the calls at any time. The support person kept a log of all telephone contacts documenting the length of each call, the woman's smoking status, issues discussed, and plans for the next contact. The first author reviewed the logs monthly and made quality control checks to verify contacts.

Participants were assessed by the nurse interviewer at the beginning of their first, second, third, fourth, and end of pregnancy (28-34 weeks) prenatal visits to determine smoking status. Self reported abstinence was defined as no smoking in the past seven days. ${ }^{6}$ 
For women who missed their end of pregnancy appointment, the interviewer attempted to reach them by telephone. Urine was collected for cotinine analysis by immunoassay at the end of pregnancy assessment to confirm smoking status; values $<80 \mathrm{ng} / \mathrm{ml}$ were considered indicative of abstinence.

\section{Results}

Baseline comparisons of women in the experimental and control conditions revealed no significant differences in demographics, pregnancy history, or smoking information (table 1).

A $\chi^{2}$ analysis examined the relation between condition and abstinence (defined by self report plus cotinine verification) at the end of pregnancy assessment. This intention to treat analysis included all 151 women and counted those lost to follow up as smokers. The analysis revealed that 14 women in the experimental condition (18.2\%) and 11 women in the comparison condition (14.9\%) were verified as having quit smoking, a non-significant relation. Of the 151 women enrolled, $16(10.6 \%)$ could not be reached for the end of pregnancy assessment, with no significant difference between conditions. Baseline comparisons between the women lost to follow up and those reached revealed only one significant difference. Women lost to follow up had a significantly lower mean education level (10.4) compared to women assessed at the end of pregnancy (11.9) $(p=0.03)$. Analyses based on data from the 135 women reached for the end of pregnancy assessment revealed no significant differences between experimental and control participants, respectively, on abstinence $(19 \% v$ $17 \%)$, reduction in smoking of greater than $50 \%$ from first prenatal visit $(42 \% v 44 \%)$, or on advancement in stage of change $(31 \% v$ $21 \%)($ all $\mathrm{p}>0.1)$.

\section{Discussion}

Our results revealed a non-significant association between condition and abstinence at the end of pregnancy, although quit rates were in the predicted direction, and the confirmed abstinence rate in the experimental condition $(18.2 \%)$ was consistent with some of the best outcomes observed in smoking cessa-

Table 1 Demographic, pregnancy, and smoking history information at first prenatal visit *

\begin{tabular}{|c|c|c|}
\hline & $\begin{array}{l}\text { Experimental } \\
\text { condition }(n=77)\end{array}$ & $\begin{array}{l}\text { Comparison } \\
\text { condition }(n=74)\end{array}$ \\
\hline \multicolumn{3}{|l|}{ Demographics } \\
\hline Age (years) (mean (SD)) & $23.1(5.6)$ & $23.7(6.7)$ \\
\hline Education (years) (mean (SD)) & $11.7(2.0)$ & $11.5(2.1)$ \\
\hline Race (white) (n (\%)) & $73(94.8)$ & $71 \quad(96.0)$ \\
\hline Ethnicity (non-Hispanic) (n (\%)) & $74 \quad(98.7)$ & 73 (98.7) \\
\hline Insurance (Medicaid) (n (\%)) & $(77.5)$ & $(74.6)$ \\
\hline \multicolumn{3}{|l|}{ Pregnancy history } \\
\hline Weeks pregnant (mean (SD)) & $11.6(5.5)$ & $11.6(5.0)$ \\
\hline Primigravida (n (\%)) & $37 \quad(48.7)$ & $30 \quad(41.7)$ \\
\hline Prior births (mean (SD)) & $0.9(1.2)$ & $1.0(1.2)$ \\
\hline \multicolumn{3}{|l|}{ Smoking information } \\
\hline Age started (years) (mean (SD)) & $14.1(3.4)$ & $14.5(2.8)$ \\
\hline Cigs/day before pregnancy (mean (SD)) & $22.6(11.3)$ & $20.2(10.1)$ \\
\hline Cigs/day at first prenatal visit (mean (SD)) & $10.5(9.6)$ & $9.8(7.8)$ \\
\hline Exhaled CO (ppm) (mean (SD)) & $11.3(7.9)$ & $11.3(8.7)$ \\
\hline Prior quit attempts (mean (SD)) & $2.6(6.5)$ & $1.5(2.7)$ \\
\hline Other smokers in household (mean (SD)) & $1.3(1.9)$ & $1.5(1.9)$ \\
\hline Intention to quit during pregnancy (n (\%)) & $57 \quad(75.0)$ & 53 (74.7) \\
\hline Intention to quit in next month (n (\%)) & $39 \quad(51.3)$ & $38 \quad(52.8)$ \\
\hline
\end{tabular}

^No significant differences were found between conditions at baseline; ppm, parts per million. tion trials conducted with pregnant women. ${ }^{1}$ Unfortunately, the number of women enrolled was approximately half that required based on the initial power calculations; therefore, these findings are inconclusive. Low enrolment was associated with a reduction in the number of pregnant women on Medicaid who sought care from this obstetric practice compared to census data four years earlier. Because smoking is more heavily concentrated among lower income women, the loss of some of the Medicaid population resulted in fewer eligible women for this study. However, among the lower income women seen within this practice, $37.1 \%$ smoked.

The confirmed abstinence rate at the end of pregnancy in our comparison condition, $14.9 \%$, is one of the highest observed in a control condition in smoking cessation studies with pregnant women, ${ }^{1}$ and was higher than we anticipated when we conducted our initial power calculations. In retrospect, it is likely that we delivered a more intensive intervention in our comparison condition than "best practice" guidelines require, as we prompted providers to address the smoking issue at each of the first three prenatal visits. Thus, we may have diminished our ability to detect an effect for the telephone support because we compared it to a second experimental condition rather than a more traditional control condition.

Our study was an effectiveness trial, and our analyses were based on intention to treat, regardless of exposure to the telephone peer support. Of the 77 women randomised into the experimental condition, $9(12 \%)$ had low intentions of quitting smoking during pregnancy and were never offered the peer support, $9(12 \%)$ had no home telephone and were not referred, and $15(19 \%)$ refused the offering, leaving $44(57 \%)$ who were referred for peer support. Data from log sheets completed by the telephone support person revealed that three women referred were never reached; therefore, only $53 \%$ of the women in the experimental condition received the peer support intervention. This lack of exposure threatened the study's internal validity, making the findings difficult to interpret.

Further examination of the telephone support logs of women referred for peer support revealed that women received a mean of 13 support contacts (range $0-32$ calls). Of the women reached, $32 \%$ reported they were abstinent at their last telephone contact, and $43 \%$ reported making a quit attempt during the support period. These data reveal more quit attempt activity than was reflected in the outcome data. We also know from follow up interviews with 19 women who received the peer support (nine had quit smoking and 10 had not) that $89 \%$ appraised the telephone contacts positively and considered them useful in helping them change their smoking. Thus, while the outcome data are inconclusive, the findings are sufficiently promising to warrant further investigation of the use of proactive telephone peer support to help pregnant women stop smoking. 
This research was supported by a grant from the Robert Wood Johnson Foundation as part of their Smoke-Free Families Program.

1 Dolan-Mullen P, Ramirez G, Groff J. A meta-analysis of randomized trials of prenatal smoking cessation interventions. Am f Obstet Gynecol 1994;171:1328-34.

2 Kottke T, Battista R, DeFreise G, et al. Attributes of successful smoking cessation interventions in medical practice: a meta-analysis of 39 controlled trials. $\mathcal{F} A M A$ 1988;259:2883-9.
3 Solomon LJ, Secker-Walker RH, Flynn BS, et al. Proactive peer support by telephone to help women quit smoking. peer support by telephone to help
Health Educ Res 1996;11:377-81.

4 Lichtenstein E, Glasgow R, Lando H, et al. Telephone counseling for smoking cessation: Rationales and meta-analytic review of evidence. Health Educ Res 1996;11:243-57.

5 Fiore M, Bailey W, Cohen S, et al. The Agency for Health Care Policy and Research Smoking Cessation clinical practice guideline. $\mathcal{F} A M A$ 1996;275:1270-80.

6 Ossip-Klein DJ, Bigelow G, Parker SR, et al. Task force 1: classification and assessment of smoking behavior. Health Psychol 1986;5(suppl):3-11.

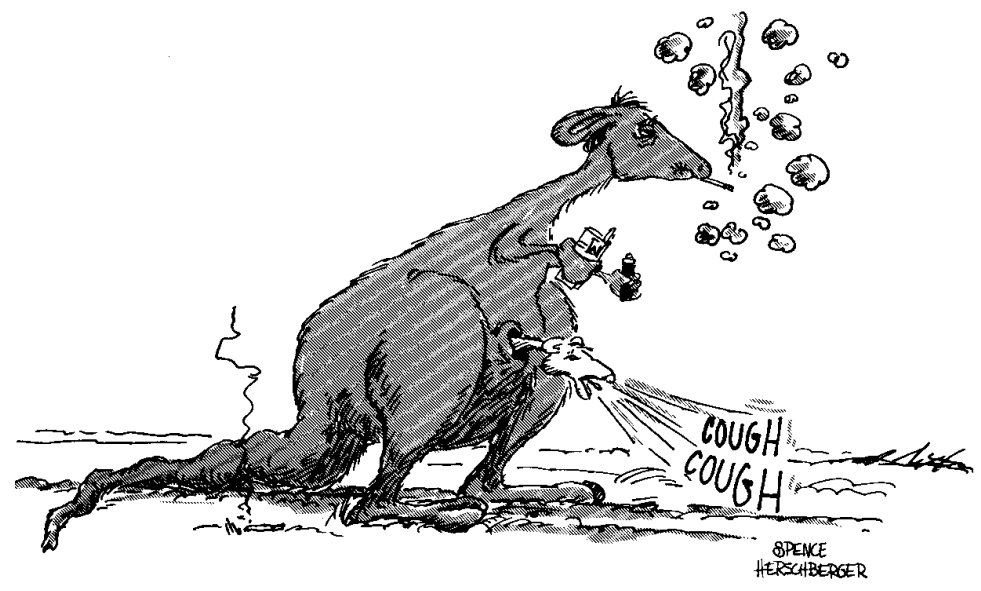

By WR Spence and V Herschberger, reprinted with permission of HEALTH EDCO, INC, Waco, Texas, USA. 